Gynäkologe 2012 · 45:260-262

DOI 10.1007/s00129-011-2888-2

(c) Springer-Verlag 2012

M. Thill ${ }^{1} \cdot$ K. Diedrich ${ }^{1} \cdot$ R. Kreienberg ${ }^{2} \cdot$ W. Jonat ${ }^{3}$

${ }^{1}$ Klinik für Frauenheilkunde und Geburtshilfe, Universitätsklinikum Schleswig-Holstein, Campus Lübeck

2 Universitätsfrauenklinik, Universität Ulm

${ }^{3}$ Klinik für Gynäkologie und Geburtshilfe, Universitätsklinikum Schleswig-Holstein, Campus Kiel

\title{
Das nichtinvasive Karzinom in der Frauenheilkunde
}

nings hat sich die Anzahl der prämalignen Vorläuferläsionen deutlich vermehrt, die Anzahl an duktalen Carcinomata in situ (DCIS) hat sich sogar verzehnfacht. Daher liegt der Anteil des DCIS mittlerweile bei etwa $20 \%$ der an einem Brustzentrum diagnostizierten Primärfälle. Unklar ist zu Zeit, ob die zunehmende Anzahl an Operationen von In-situ-Karzinomen zu einer Reduktion der invasiven Karzinome führt, oder ob sie lediglich eine Übertherapie bedeutet.

\section{》) Aufgrund der Ab- \\ klärungsdiagnostik sind wir vermehrt mit prämalignen Läsionen konfrontiert}

Das vorliegende Heft befasst sich eingehend und sehr ausführlich mit den In-situ-Karzinomen von Mamma, Zervix, Vulva und Vagina sowie mit dem BorderlineTumor des Ovars. Darüber hinaus gibt es einen Überblick über die entsprechende Diagnostik sowie ihren jeweiligen Stellenwert und stellt sowohl Standards als auch aktuelle Entwicklungen dar.

Welche Diagnostik beim DCIS zum Standard gehört und welche innovativen Techniken noch in der Entwicklung stecken zeigen Schem u. Schäfer auf. Neben der Diskussion um den Stellenwert der Magnetresonanztomographie (MRT) werden auch Tomosynthese und Elasto- graphie in einen aktuellen Kontext gesetzt. Die operativen, lokalen und systemischen Therapieoptionen werden von Thill et al. ausführlich und kritisch erörtert. Bedingt durch den Anstieg der prämalignen und malignen Vorläuferläsionen der Brust richten die Autoren ihr Augenmerk auch auf neue intraoperative Tools zur Detektion der Läsionen und zur Schnittrandbeurteilung.

\section{) Immer noch wird die HPV-Impfung noch nicht hinreichend genutzt}

Sörgel et al. befassen sich mit dem Management der zervikalen intraepithelialen Neoplasie (CIN) III. Obwohl eine HPV-Impfung zur Verfügung steht, ist das Impfverhalten der jungen Frauen noch immer mehr als unbefriedigend. In der Folge bleibt die Rate an Konisationen hoch und das Frühgeburtlichkeitsrisiko durch eine Konisation mit konsekutiver Zervixinsuffizienz bestehen. Hoellen et al. plädieren daher für den gezielten Einsatz der Kolposkopie, um die Rate an Konisationen zu senken. In ihrem Beitrag zeigen die Autoren auf, dass es das Ziel der Kolposkopie ist, zum einen die Anzahl der übersehenen Karzinome zu reduzieren, zum anderen eine Übertherapie bei auffälligem Pap-Abstrich im zu vermeiden. 
Hier steht eine Anzeige.

算 Springer 
Anfangs belächelt und schließlich mit dem Nobelpreis ausgezeichnet - durch seinen hartnäckigen Forschergeist hat Prof. Harald zur Hausen bewiesen, dass das humane Papillomavirus (HPV) für die Entstehung des Zervixkarzinoms verantwortlich ist. Mittlerweile gibt es genügend Daten, die dafür sprechen, dass auch Malignome der Vulva und der Vagina mit HPV assoziiert sind. Beyer et al. beschäftigen sich daher mit der Rolle des HPV in der Gynäkoonkologie und setzen sich kritisch mit der polarisierend geführten Diskussion um das Für und Wider der HPVImpfung auseinander.

\section{》) Medizinisch und psychoonkologisch müssen wir uns mit der steigenden VIN-Inzidenz auseinandersetzen}

Jentschke et al. widmen sich der Behandlung der vulvären und vaginalen intraepithelialen Neoplasie (VIN/VaIN) III und gehen ausführlich auf die verschiedenen Therapieoptionen ein. Gerade vor dem Hintergrund der in den letzten Jahren bei jungen Frauen angestiegenen VIN-III-Erkrankungen ist dies ein Thema, mit dem wir medizinisch und psychoonkologisch konfrontiert sind. Man wird sehen, welchen Einfluss die HPV-Impfung haben wird und ob sich diese Entwicklung dadurch umkehren lässt.

Schließlich befassen sich Hilpert et al. mit einem Tumor des Ovars, der in den letzten Jahren immer wieder für Diskussionsstoff gesorgt hat, mit dem Borderline-Tumor. Die Auswertungen histologisch zentral nachbefundeter Borderline-Tumoren haben uns gezeigt, wie nah Unter- oder Übertherapie beieinanderliegen, wenn Ovarialkarzinome übersehen oder Borderline-Tumoren aggressiv systemisch therapiert werden.

Wir sind sicher, Ihnen, mit dem in diesem Heft behandelten Leitthema, zahlreiche Informationen an die Hand geben zu können, die Sie in Ihrer Praxis und in Ihrem Arbeitsalltag zur Optimierung Ihrer diagnostischen und therapeutischen Algorithmen verwenden können und wünschen Ihnen viel Spaß bei der Lektüre.

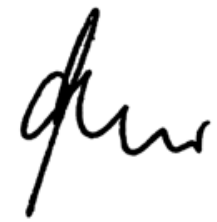

PD Dr. M. Thill

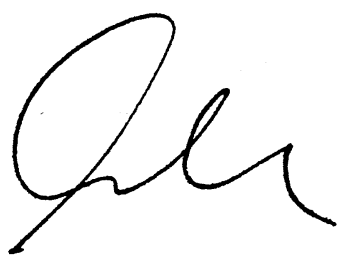

Prof. Dr. K. Diedrich
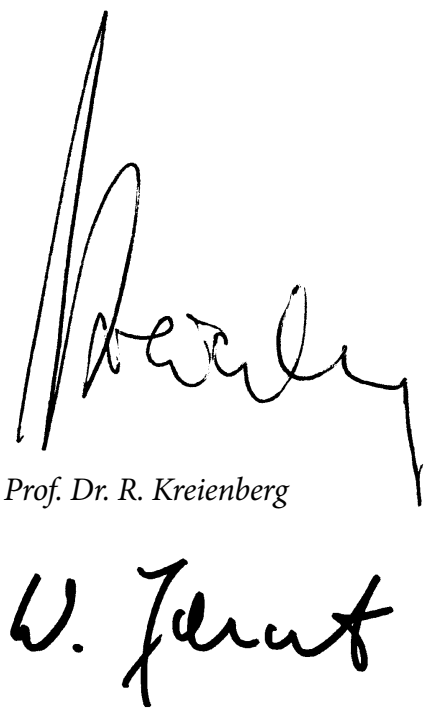

Prof. Dr. W. Jonat

\section{Korrespondenzadresse}

\section{PD Dr. M. Thill}

Klinik für Frauenheilkunde und Geburtshilfe, Universitätsklinikum Schleswig-Holstein, Campus Lübeck

Ratzeburger Allee 160, 23538 Lübeck

marc.thill@uk-sh.de

Prof. Dr. K. Diedrich

Klinik für Frauenheilkunde und Geburtshilfe, Universitätsklinikum Schleswig-Holstein, Campus Lübeck

Ratzeburger Allee 160, 23538 Lübeck

klaus.diedrich@uk-sh.de

\section{Das Ende des „,Maiglöckchen-} Phänomens"

Spermien haben einen weiten Weg vor sich, um die Eizelle zu finden; nur wenige der Millionen von Spermien erreichen ihr Ziel. Durch Chemotaxis unterstützt die Eizelle die Spermien bei ihrer Suche, indem sie Lockstoffe aussendet, die den Kalziumhaushalt der Spermienzellen beeinflusst. Es wurden verschiedene Konzepte veröffentlicht, wie menschliche Spermien die Eizelle aufspüren. Eine 2011 veröffentlichte Arbeit schlägt vor, dass das weibliche Sexualhormon Progesteron - das von Kumuluszellen in der Nähe der Eizelle gebildet wird - die Spermien anlockt. Für die Progesteronwirkung sind sogenannte CatSperIonenkanäle (cation channels of sperm) verantwortlich. Die CatSper-Kanäle, die sich ausschließlich in Spermien befinden, sind unverzichtbar für die Fortpflanzung. Männer, die einen Gendefekt für CatSper tragen, sind unfruchtbar. Die Wissenschaftler konnten zeigen, dass Progesteron die CatSper-Kanäle direkt öffnet, wodurch Kalzium in die Spermien-Zelle strömt. In einer aktuellen Studie, berichten die Forscher nun, dass Bourgeonal, ein Bestandteil des Maiglöckchenduft, der in einem anderen Modell als Lockstoff vorgeschlagen wurde, die Progesteronwirkung auf Spermien imitiert. Er öffnet die CatSper- Kanäle ebenfalls direkt. Allerdings wirken die Duftstoffe erst bei mehr als 1000-fach höheren Konzentrationen als Progesteron! Die Bonner Forscher konzentrieren sich jetzt darauf, neben Progesteron weitere Lockstoffe im Eileiter zu identifizieren. Wenn es gelänge, die Wirkung weiblicher Faktoren auf die CatSper-Kanäle zu stören, könnte das zu einem neuartigen Verhütungsmittel - der Pille für den Mann - führen. Bis dahin ist es allerdings noch ein langer Weg.

Literatur: Brenker, C., Goodwin, N., Weyand, I. et al (2012) The CatSper channel: a polymodal chemosensor in human sperm. EMBO J e-pub ahead of print doi: 10.1038/ emboj.2012.30.

Quelle: Center of advanced european studies and research, $h t t p: / / w w w . c a e s a r . d e$ 